Marquette University

e-Publications@Marquette

College of Education Faculty Research and

Publications

Education, College of

4-10-2018

Polycystic Ovary Syndrome Across the Life Span of Women: A Wellness-Based Integrative Intervention

Karisse A. Callender

Marquette University, karisse.callender@marquette.edu

Accepted version. Adultspan Journal, Vol.17, No. 1 (April 2018): 41-52. DOI. (C) 2018 John Wiley \& Sons, Inc. Used with permission. 
Marquette University

\section{e-Publications@Marquette}

\section{Education Faculty Research and Publications/College of Education}

This paper is NOT THE PUBLISHED VERSION; but the author's final, peer-reviewed manuscript.

The published version may be accessed by following the link in the citation below.

Adultspan Journal, Vol. 17, No. 1 (April, 2018): 41-52. DOI. This article is (C) Wiley and permission has been granted for this version to appear in e-Publications@Marquette. Wiley does not grant permission for this article to be further copied/distributed or hosted elsewhere without the express permission from Wiley.

\section{Contents}

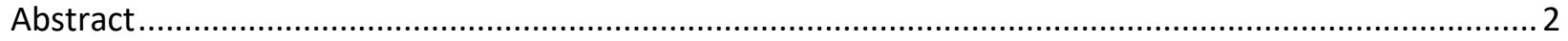

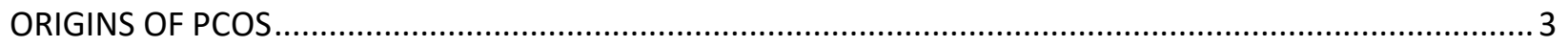

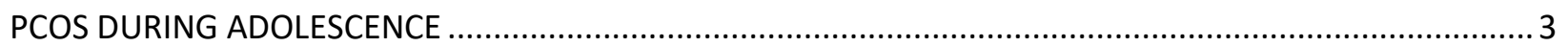

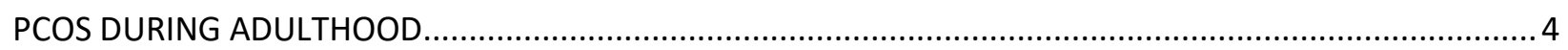

COUNSELING INTERVENTIONS FOR WOMEN WITH PCOS ............................................................ 4

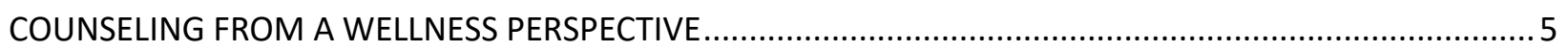

A WELLNESS - BASED INTEGRATIVE INTERVENTION FOR PCOS …................................................... 5

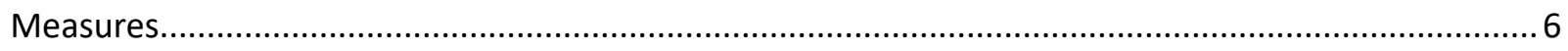

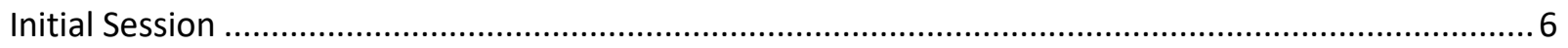

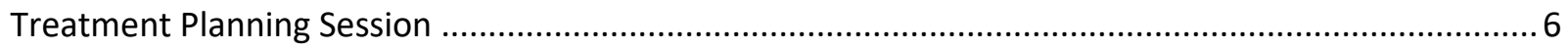

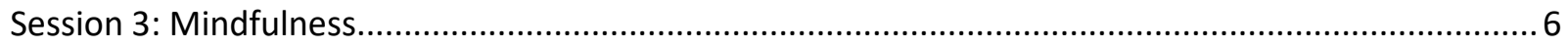

Sessions 4 and 5: Identifying Thought and Behavior Patterns Related to PCOS ................................. 7

Sessions 6 and 7: Learning About Emotions While Living With PCOS .............................................. 7

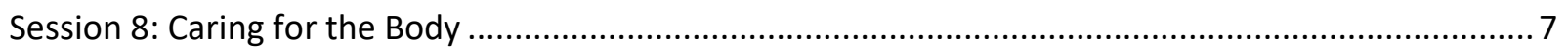

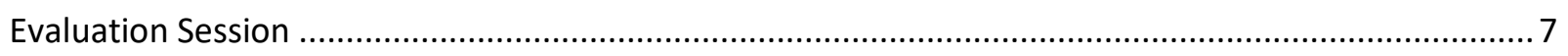

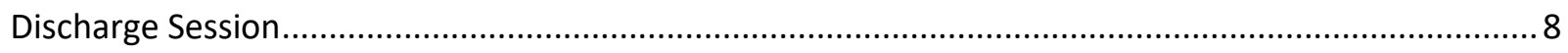

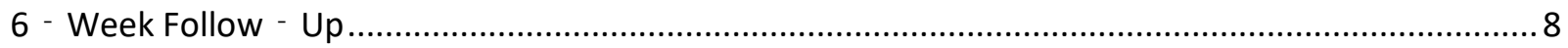




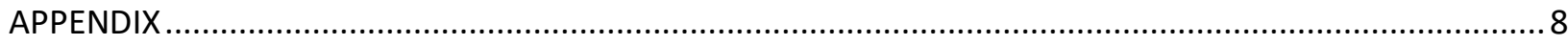

A Wellness - Based Integrative Intervention for Polycystic Ovary Syndrome (PCOS) .......................... 8

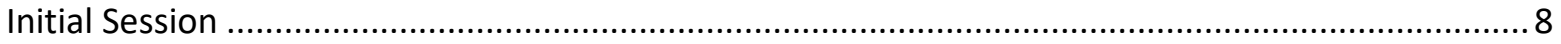

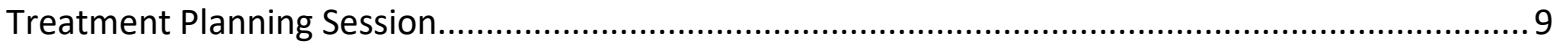

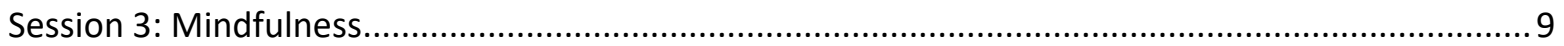

Sessions 4 and 5: Identifying Thought and Behavior Patterns Related to PCOS .............................. 9

Sessions 6 and 7: Learning About Emotions While Living With PCOS ............................................ 9

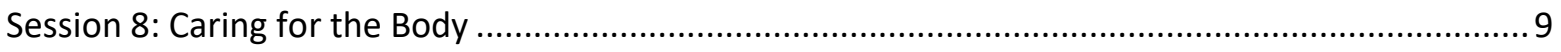

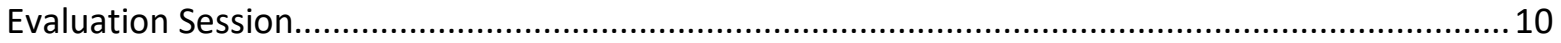

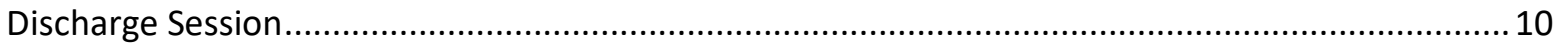

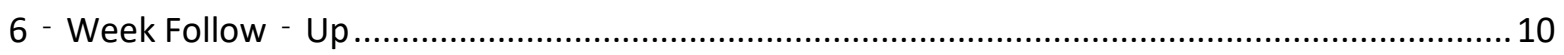

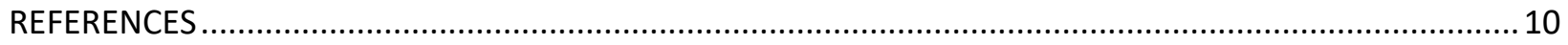

\title{
Polycystic Ovary Syndrome Across the Life Span of Women: A Wellness-Based Integrative Intervention
}

\author{
Karisse A. Callender \\ Marquette University, Milwaukee, WI
}

\begin{abstract}
Women diagnosed with polycystic ovary syndrome (PCOS) - an endocrine disorder that affects a woman's physical and psychological functioning-face challenges that affect personal and social functioning. Information about PCOS is provided for counselors, including a description of a wellnessbased integrative intervention to enhance optimal wellness and quality of life.

Polycystic ovary syndrome (PCOS) is considered the most common endocrine disorder in women, affecting between an estimated $4 \%$ and $12 \%$ of women of reproductive age (Smith \& Taylor, 2011). There are several physical characteristics associated with PCOS that may negatively impact the mental health of women diagnosed with this condition. Clinical manifestations of PCOS include obesity, infertility, acne, male-pattern hair growth (hirsutism), hair loss (alopecia), increased hormone level,
\end{abstract}


irregular menstrual cycle, decreased fertility, and negative impacts on cardiovascular health (Froeschle, Castillo, Mayorga, \& Hargrave, 2008). In addition, women can experience high levels of the male hormone androgen and multiple small cysts on their ovaries. Women diagnosed with PCOS are also at increased risk for mental health problems, such as depression, anxiety, and eating disorders (Annagur, Kerimoglu, Tazegul, Gunduz, \& Gencoglu, 2015).

\section{ORIGINS OF PCOS}

Stein Leventhal first diagnosed PCOS in 1935 after conducting a study on seven women who exhibited multiple cysts within their ovaries accompanied by a variety of symptoms (Froeschle et al., 2008). The name of this syndrome can be misleading because cystic ovaries are not a necessary symptom for diagnosis (Farkas, Rigo, \& Demetrovics, 2014; Tehrani, 2015). Although the etiology of PCOS has been unclear in the past, advances in research have increased and clarified the amount of information available both to individuals diagnosed with PCOS and providers involved in treatment. Early signs of PCOS often begin during fetal development and remain dormant during childhood. The most common underlying mechanism linked to PCOS is believed to be insulin resistance, a chronic and progressive disturbance of metabolic function, which explains the obesity and other metabolic disorders associated with PCOS (Dowdy, 2012).

Although PCOS is classified as an endocrine disorder with several physical clinical manifestations, research indicates that there is an increased risk for mood disorders, such as depression and anxiety (Annagur et al., 2015; Dowdy, 2012; Farkas et al., 2014). Therefore, counselors should be aware of the psychological factors associated with PCOS and how these factors affect the lives of women. Counselors have the unique opportunity to assist and empower these women as they learn to strive for optimal wellness and navigate the challenges associated with a PCOS diagnosis.

\section{PCOS DURING ADOLESCENCE}

Because PCOS typically manifests during adolescence, during this time, girls may present with irregularities in menstruation, signs of obesity, Type 2 diabetes, hirsutism, and acne (Dowdy, 2012). PCOS may be undiagnosed during early adolescence, especially at the onset of the menstrual cycle when oral contraceptives may be prescribed to treat adolescents for menstrual irregularity, acne, or hirsutism (Snyder, 2005). Adolescent girls diagnosed with PCOS may experience a lower quality of life, especially within social and emotional dimensions (Jones, Hall, Lashen, Balen, \& Ledger, 2011).

A study conducted by Laggari et al. (2009), with 49 adolescent girls, found that adolescent girls with PCOS showed higher anxiety scale scores than did a control group of adolescent girls without PCOS. Other mental health issues reported included feeling depressed, pessimistic, irritable, and upset (Jones et al., 2011; Rofey et al., 2009). A fear of possible infertility may also affect quality of life in adolescent girls (Lee, 2015). Another issue for adolescent girls is the increased likelihood of engaging in risky sexual behavior due to a mistaken belief that pregnancy is impossible because of PCOS (Lee, 2015). Some adolescent girls may also develop eating disorders to cope with weight gain, including bingeing, purging, and excessive dieting. Failure to lose weight may cause feelings of distress and inadequacy, leading to isolation and unhealthy eating patterns (Lee, 2015).

Hirsutism, or abnormal male-pattern hair growth, is another psychologically distressing occurrence for adolescent girls that can be humiliating, especially when hair growth is on the face (Froeschle et al., 
2008). Excess facial hair can be in large areas that may appear beard-like and prompt regular shaving. Girls may also develop thick sideburns, hair over the upper lips, and thick eyebrows. Adolescent girls with PCOS reported being afraid of excess hair, feeling unfeminine, and experiencing social isolation (Dowdy, 2012). This feeling of unattractiveness can increase feelings of shame and rejection and affect self-perception and relationships with family and friends. In addition, changes in mood may rupture personal and social relationships, resulting in increased isolation (Jones et al., 2011).

\section{PCOS DURING ADULTHOOD}

Adult women experience concerns like those reported by adolescents with PCOS. Castillo (2008) identified several themes about the social and emotional experiences of women diagnosed with PCOS through a qualitative study. Women diagnosed with PCOS report a variety of concerns that begin in adolescence and continue across the life span. These include social insecurity, low self-esteem, feelings of helplessness, strained relationships, and mood disorders. The chronic condition of PCOS produces metabolic and pregnancy complications, infertility, obesity, reduced quality of life, mood disorders, and decreased self-worth (Garad, Teede, \& Moran, 2011). For a woman considering starting a family, infertility can be a stressful and life-changing experience (Galhardo, Pinto-Gouveia, Cunha, \& Matos, 2011). Some women are first diagnosed with PCOS after seeking treatment for infertility. Infertility can also affect romantic relationships because partners' perceptions of their personal quality of life is influenced by their shared and individual experiences (Dennett \& Simon, 2015). The impact of infertility may be profound, resulting in psychological distress and threats to the identity of being a woman. Although a significant proportion of women diagnosed with PCOS are infertile, a modest reduction in weight (5\%) may result in improved reproductive function, menstrual regularity, and higher ovulation rates (Mahoney, 2014).

\section{COUNSELING INTERVENTIONS FOR WOMEN WITH PCOS}

Interventions for PCOS typically focus on the treatment of physical symptoms (Annagur, Tazegul, \& Akbaba, 2014). However, interventions focused on improving psychological well-being should encourage women diagnosed with PCOS to take a proactive role in their own physical and mental health. This may lead to a more holistic lifestyle and reduced effects from disease and other ailments (Fetter \& Koch, 2009). Interventions may include techniques from cognitive behavior therapy (CBT), dialectical behavior therapy (DBT), and mindfulness.

The proposed integrated intervention incorporates techniques used in $\mathrm{CBT}, \mathrm{DBT}$, and mindfulness. $\mathrm{CBT}$ and DBT have recorded success in treating various disorders such as major depressive disorder, generalized anxiety, health anxiety, eating disorders, obesity, and mood dysregulation (Beck, 2011; Linehan, 2015). Counseling professionals who use these interventions help clients to target unhealthy thinking patterns and change behaviors and interpersonal patterns that influence and, ultimately, maintain problems in living (Linehan, 2015). However, it is recommended that counselors approach sessions within a holistic and wellness-based framework and integrate mindfulness into counseling sessions. Mindfulness is a way for clients to cultivate clarity, awareness, and acceptance of the present moment (Kabat-Zinn, 2005). Mindfulness skills include the intentional process of observing, describing, and participating in the moment effectively and without judgment (Hayes, Follette, \& Linehan, 2004). The integration of these techniques can help to target the most important psychological concerns of women diagnosed with PCOS. 


\section{COUNSELING FROM A WELLNESS PERSPECTIVE}

Counselors assist and encourage clients to strive toward their own definition of wellness using a developmental approach. Clients are encouraged to seek independence from the helping relationship through transferable, generalizable skills attained in counseling sessions (Eaves, Erford, \& Fallon, 2010). From a wellness perspective, counseling takes a more holistic approach that integrates physical, emotional, spiritual, mental, and social factors of the individual's life. Wellness counselors believe that human potential, personal strengths, and lifestyle are strong indicators of well-being. Therefore, wellness counselors help clients to strive for their highest level of functioning across all dimensions of life (Granello \& Witmer, 2013).

Because PCOS negatively influences the moods of women with the diagnosis (Annagur et al., 2015; Farkas et al., 2014; Lee, 2015), it is important to assist women with emotion regulation as a strategy for achieving a more balanced lifestyle. Hunnicutt-Hollenbaugh (2013) discussed the relationship between wellness and emotion regulation, stating that improvements in one area of an individual's life can positively affect other dimensions as well. Essentially, improvements in emotion regulation can lead to improvements in one's physical state.

Counselors working in diverse settings such as schools, community agencies, and private practice may encounter adolescent girls and women diagnosed with PCOS who experience anxiety and depression. Counselors can help clients focus on wellness and lifestyle change to mitigate the effects of PCOS by changing thoughts, behaviors, and attitudes toward themselves and others. Yoga, meditation, exercise, and mindfulness practices may serve as useful adjunct treatments alongside other counseling interventions. Wellness counseling can also serve as a platform for women diagnosed with PCOS to learn about the importance of self-care in relation to physical health (Elliot, 2013). Sweeney and Myers (2004) suggested four steps to wellness counseling: (a) introduction of a wellness model, including a life-span focus; (b) assessments that can be formal or informal, based on the model; (c) interventions based on selected areas to enhance wellness; and (d) evaluation, follow-up, and repetition of the second and third steps, if necessary.

\section{A WELLNESS-BASED INTEGRATIVE INTERVENTION FOR PCOS}

Treatment planning for women diagnosed with PCOS includes targeting physical and psychological symptoms. Intensive changes in lifestyle via behavioral interventions are considered the first-line interventions for symptom management (Dennett \& Simon, 2015). Counseling professionals must recognize the psychological factors associated with PCOS to effectively assist women in improving their quality of life via psychoeducation and behavior change (Benson et al., 2010). The Wellness-Based Integrative Intervention (Wel-BII) is a psychoeducational program for women diagnosed with PCOS based on the principles of CBT, DBT, mindfulness, and wellness counseling. I selected these interventions based on their strong focus on behavior and lifestyle change, ultimately targeting psychological and physical symptom management.

Wel-BII is a 12-week intervention with a 6-week follow-up contact. The intervention is focused on cognitive restructuring, behavior modification, and acceptance through assessment, planning, education, evaluation, and follow-up. Wel-Bll can be used with adolescent girls and women diagnosed with PCOS. In addition to the foundational principles of CBT, DBT, mindfulness, and wellness interventions, Wel-BII focuses specifically on the characteristic features of PCOS to help women learn to 
modify thoughts and feelings about themselves in relation to being diagnosed and living with PCOS. Wel-BII is composed of 12 sessions, each 60 minutes in length, with an electronic follow-up (see Appendix):

- 1-hour initial session

- 1-hour treatment planning session

- Eight 1-hour sessions with structured interventions

- 1-hour evaluation session

- 1-hour discharge session

- 6-week follow-up

\section{Measures}

Measures used in the Wel-BII include the Five Factor Wellness Inventory (5F-WEL; Myers \& Sweeney, 2005) and the Polycystic Ovary Syndrome Questionnaire (PCOSQ; Cronin et al., 1998). The 5F-WEL is a 91-item, self-scoring, evidence-based tool used to evaluate the characteristics of wellness. There are 17 discrete scales, with responses ranging from strongly disagree to strongly agree. The reliability score for total wellness is .94, and the measure was normed on an adult population. The PCOSQ is a 26-item instrument measuring five areas-emotions, body hair, weight, infertility problems, and menstrual problems-on a 7-point scale. The dimensions had Cronbach's alpha scores ranging from .70 to .97 (Jones et al., 2004).

\section{Initial Session}

In the first session, the counselor introduces Myers, Sweeney, and Witmer's (2000) Wheel of Wellness with an explanation of the benefits of healthy living on global well-being and also conducts a mood check. A copy of the model is shared, and an explanation of each characteristic is described. Clients are encouraged to provide a personal definition of wellness and to discuss goals for personal wellness with the counselor (Myers \& Sweeney, 2005). Copies of the 5F-WEL (Myers \& Sweeney, 2005) and the PCOSQ (Cronin et al., 1998) are given as assessment instruments to be self-administered at home, and discussed in the second session with the counselor. The counselor assigns an action plan to identify an image that represents wellness to be reviewed in the following treatment planning session. An action plan, formerly known as "homework" in CBT, includes activities that clients complete between sessions, without the presence of the therapist (Beck, 2011).

\section{Treatment Planning Session}

During the second session, the counselor conducts a mood check, reviews the image of wellness, and reviews the results of the 5F-WEL and PCOSQ. The counselor conducts a clinical assessment and discusses the client's expectations for treatment (Beck, 2011). The counselor collaborates with the client to develop a treatment plan based on the PCOSQ scores and areas of need on the 5F-WEL. The client is encouraged to think about how each dimension of her life has been affected by the PCOS diagnosis, along with desired changes (Myers \& Sweeney, 2005). The counselor assigns a wellness plan that requires clients to write a list of thoughts about self in relation to being diagnosed with PCOS.

\section{Session 3: Mindfulness}

The client begins this session by completing a mood check and reviewing the action plan assignment with the counselor. Next, the counselor introduces the concept of mindfulness, discusses how it may be 
helpful for women with PCOS, and explains the benefits of mindfulness and acceptance to lifestyle changes. The counselor describes various types of mindfulness activities and how these activities can be incorporated into daily living activities. Suggested mindfulness activities include mindful eating, meditation, deep breathing, and journaling. The client is asked to create a list of personally selected mindfulness activities, which will be included in her action plan.

\section{Sessions 4 and 5: Identifying Thought and Behavior Patterns Related to PCOS}

In the fourth session, the counselor begins with a mood check followed by a mindfulness activity. Next, the counselor and client begin working on the treatment plan objectives. The client learns to identify negative thoughts about self and recognize behaviors that support the negative thoughts. In Session 4, the counselor reviews the action plan from the previous session and introduces a thought log. The client is assigned a thought log as an action plan to prepare for Session 5. In Session 5, the client engages in a mood check followed by a mindfulness activity. Next, the client learns to challenge negative thoughts, learn healthier thought patterns, and change unhealthy behavior patterns. The client is given a behavior checklist as an action plan to prepare for Session 6.

\section{Sessions 6 and 7: Learning About Emotions While Living With PCOS}

Session 6 begins with a mood check and mindfulness activity, a review of the behavior checklist, and an introduction to emotions as they relate to PCOS. The client is educated on the purpose of emotions, how to understand emotions, and strategies for identifying emotions (Linehan, 2015). To prepare for Session 7, the client is asked to keep an emotion log each day. Session 7 begins with a mood check, a mindfulness activity, and a review of the emotion log. Next, the counselor educates the client on strategies for managing emotions during difficult times. For the action plan, the client makes a list of physical activities she enjoys or would like to participate in, to be reviewed next session.

\section{Session 8: Caring for the Body}

After the mood check and mindfulness activity, the counselor reviews the client's list of physical activities and discusses realistic options to increase her level of exercise and improve nutrition. The client is educated on the benefits of exercise and nutrition in the treatment and management of PCOS, including the benefits of maintaining contact with her primary care physician for continued health monitoring and medication management. The counselor also educates the client on the benefits of meditation, yoga, and other mindfulness activities for health benefits. The action plan assignment includes a written reflection about the past 10 weeks to be discussed next session. In addition, the client will be asked to complete another PCOSQ to be reviewed next session.

\section{Evaluation Session}

The counselor begins the evaluation session with a mood check and mindfulness activity, reviews the written reflection with client, and discusses any concerns or questions. Next, the counselor and client review the results of the PCOSQ and compare them with the initial results. The counselor then reviews the treatment plan and, in collaboration with the client, evaluates the need for further sessions. If the client is satisfied with the progress made, the counselor discusses discharge planning and recommends termination at the next session. If discharge is the plan, the client is asked to create coping cards for her most challenging situations. The coping cards will consist of words or phrases written on small note cards that the client will use for self-soothing, thought reframe, and coping skills during moments of 
distress. If the decision is to develop a new treatment plan for further sessions, the client is asked to make a list of her primary concerns to assist in modifying the current plan of care.

\section{Discharge Session}

The final session begins with a mood check and mindfulness activity followed by a review of the client's personalized coping cards created for her action plan. Next, the counselor and client collaboratively develop a discharge plan focused on a relapse prevention plan, which incorporates the coping cards as a strategy for helping the client quickly and effectively reframe common negative thoughts identified throughout the course of treatment. The relapse prevention plan includes an action plan to be used when the client notices her thoughts, emotions, or behaviors are becoming unhealthy. The client is provided with copies of thought logs, behavior checklists, coping cards, and a list of resources. The client is asked to sign an agreement for contact in the next 6 weeks for follow-up via a survey distributed online or by mail.

\section{6-Week Follow-Up}

Six weeks after discharge, it is recommended that the counselor do a follow-up with the client to assess her lifestyle progress since therapy. This follow-up is essential to monitor the impact of lifestylesustaining behaviors that were taught and implemented during therapy. The counselor may contact the client via e-mail, with a link to an online assessment, or by sending a printed assessment in the mail, to be filled out and returned to the counselor by mail. This assessment should include the PCOSQ. Based on the information provided from the PCOSQ, the client may be contacted to inquire about returning to therapy for further sessions.

\section{CONCLUSION}

PCOS is a medical condition that adversely impacts a woman's physical state. Many women also experience significant psychological effects, which can be detrimental if not addressed with appropriate treatment. Once these psychological effects are identified, counselors can provide services to help women adapt and maintain lifestyle changes while mitigating psychological distress. Counseling services, specifically wellness-based integrative counseling, can be an appropriate and effective adjuvant technique. The Wel-BII provides a treatment program designed to address the psychological concerns associated with PCOS.

\section{APPENDIX}

\section{A Wellness-Based Integrative Intervention for Polycystic Ovary Syndrome (PCOS) Initial Session}

- Introduce Myers et al.'s Wheel of Wellness

- Explain benefits of healthy living and wellness counseling

- Discuss client and counselor expectations from counseling

- Discuss client definition of wellness

- Five Factor Wellness Inventory (5F-WEL) and Polycystic Ovary Syndrome Questionnaire (PCOSQ) assessments self-administered before next session

- Action plan: Identify an image that represents wellness 
Treatment Planning Session

- Review action plan

- Review results of 5F-WEL and PCOSO

- Conduct clinical assessment of client

- Develop treatment plan based on PCOSQ score and 5F-WEL results

- Client to decide how low dimensions on 5F-WEL are affected by PCOS and what change is needed

- Action plan: Thoughts about self as related to a diagnosis of PCOS

Session 3: Mindfulness

- Review action plan

- Introduce mindfulness for women with PCOS

- Discuss benefits of mindfulness and acceptance to lifestyle change

- Discuss types of mindfulness techniques

- Action plan: Make a list of mindfulness activities that seem interesting

Sessions 4 and 5: Identifying Thought and Behavior Patterns Related to PCOS

- Session 4: Mood check and mindfulness activity

- Review action plan

- Psychoeducation on cognitive distortions

- Introduce thought log

- Action plan: Thought log

- Session 5: Mindfulness activity and mood check

- Review action plan

- Challenge negative thoughts, thought replacement

- Action plan: Behavior checklist

Sessions 6 and 7: Learning About Emotions While Living With PCOS

- Session 6: Mood check and mindfulness activity

- Review homework

- Discuss emotions as they relate to PCOS

- Action plan: Emotion log

- Session 7: Mindfulness activity and mood check

- Review action plan

- Discuss managing emotions

- Action plan: Create a list of physical activities

Session 8: Caring for the Body

- Mood check and mindfulness activity

- Review action plan

- Increase exercise and improve nutrition

- Discuss benefits of meditation, yoga, and other mindfulness activities

- Action plan: Written reflection of past 10 weeks, self-administered PCOSQ 


\section{Evaluation Session}

- Mood check and mindfulness activity

- Review action plan and PCOSQ results

- Review treatment plan and evaluate for need for further sessions

- Plan for initial discharge or revise treatment plan

- Action plan: Create coping cards for challenging situations (for discharge) or create list of primary concerns (for revised treatment plan)

\section{Discharge Session}

- Mood check and mindfulness activity

- Review action plan

- Create relapse prevention plan

- Provide client with copies of thought logs, behavior checklist, coping cards, and list of resources

- Sign agreement for client follow-up in 6 weeks via survey online or in the mail

6-Week Follow-Up

- Contact client via e-mail with link to assessment or mail a paper copy of the assessment

- Based on results, contact client to inquire about continuing counseling sessions

\section{REFERENCES}

Annagur, B. B., Kerimoglu, O. S., Tazegul, A., Gunduz, S., \& Gencoglu, B. B. (2015). Psychiatric comorbidity in women with polycystic ovary syndrome. Journal of Obstetrics and Gynaecology Research, 41, 1229-1233. doi:10.1111/jog.12696

Annagur, B. B., Tazegul, A., \& Akbaba, N. (2014). Body image, self-esteem and depressive symptomatology in women with polycystic ovary syndrome. Archives of Neuropsychiatry, 51, 129-132. doi: $10.4274 /$ npa.y6778

Beck, J. S. (2011). Cognitive behavior therapy: Basics and beyond (2nd ed.). New York, NY: Guilford Press.

Benson, S., Hahn, S., Tan, S., Janssen, O. E., Schedlowski, M., \& Elsenbruch, S. (2010). Maladaptive coping with illness in women with polycystic ovary syndrome. Journal of Obstetric, Gynecologic \& Neonatal Nursing, 39, 37-45. doi:10.1111/j.1552-6909.2009.01086.x

Castillo, Y. (2008). Understanding the social and emotional experiences of females with polycystic ovary syndrome (PCOS) (Doctoral dissertation). Retrieved from ProQuest Dissertations and Theses database. (UMI No. 3322110)

Cronin, L., Guyatt, G., Griffith, L., Wong, E., Azziz, R., Futterweit, W., ... Dunaif, A. (1998). Development of a health-related quality-of-life questionnaire (PCOSQ) for women with polycystic ovary syndrome (PCOS). Journal of Clinical Endocrinology and Metabolism, 83, 1976-1987.

Dennett, C. C., \& Simon, J. (2015). The role of polycystic ovary syndrome in reproductive and metabolic health: Overview and approaches for treatment. Diabetes Spectrum, 28, 116-120. doi: $10.2337 /$ diaspect.28.2.116

Dowdy, D. (2012). Emotional needs of teens with polycystic ovary syndrome. Journal of Pediatric Nursing, 27, 55-64. doi:10.1016/i.pedn.2010.08.001 
Eaves, S. H., Erford, B. T., \& Fallon, M. K. (2010). Becoming a professional counselor. In. B. T. Erford (Ed.), Orientation to the counseling profession: Advocacy, ethics, and essential professional foundations (pp. 3-23). Boston, MA: Pearson.

Elliot, M. (2013). Preventative self-care: Benefits of modern medicine. In P. F. Granello (Ed.), Wellness counseling (pp. 135-147). Boston, MA: Pearson.

Farkas, J., Rigo, A., \& Demetrovics, Z. (2014). Psychological aspects of the polycystic ovary syndrome. Gynecological Endocrinology, 30, 95-99. doi:10.3109/09513590.2013.852530

Fetter, H., \& Koch, D. W. (2009). Promoting overall health and wellness among clients: The relevance and role of professional counselors. Adultspan Journal, 8, 4-16. doi:10.1002/j.2161$\underline{0029.2009 . t b 00053 . x}$

Froeschle, J. G., Castillo, Y., Mayorga, M. G., \& Hargrave, T. (2008). Counseling techniques for adolescent females with polycystic ovary syndrome. Journal of Professional Counseling: Practice, Theory, and Research, 36, 17-29.

Galhardo, A., Pinto-Gouveia, J., Cunha, M., \& Matos, M. (2011). The impact of shame and self-judgment on psychopathology in infertile patients. Human Reproduction, 26, 2408-2414. doi:10.1093/humrep/der209

Garad, R., Teede, H., \& Moran, L. (2011). An evidence-based guideline for polycystic ovary syndrome. Australian Nursing Journal, 19, 30-33.

Granello, P. F., \& Witmer, J. M. (2013). Theoretical models for wellness counseling. In P. F. Granello (Ed.), Wellness counseling (pp. 29-36). Boston, MA: Pearson.

Hayes, S. C., Follette, V. M., \& Linehan, M. M. (2004). Mindfulness and acceptance: Expanding the cognitive-behavioral tradition. New York, NY: Guilford Press.

Hunnicutt-Hollenbaugh, K. M. (2013). Emotional regulation: Stones and water. In P. F. Granello (Ed.), Wellness counseling (pp. 92-103). Boston, MA: Pearson.

Jones, G. L., Benes, K., Clark, T. L., Denham, R., Holder, M. G., Haynes, T. J., ... Ledger, W. L. (2004). The Polycystic Ovary Syndrome Health-Related Quality of Life Questionnaire (PCOSQ): A validation. Human Reproduction, 19, 371-377.

Jones, G. L., Hall, J. M., Lashen, H. L., Balen, A. H., \& Ledger, W. L. (2011). Health-related quality of life among adolescents with polycystic ovary syndrome. Journal of Obstetric, Gynecologic \& Neonatal Nursing, 40, 577-588. doi:10.1111/j.1552-6909.2011.01279.x

Kabat-Zinn, J. (2005). Wherever you go, there you are: Mindfulness meditation in everyday life (10th ed.). New York, NY: Hachette Books.

Laggari, V., Diareme, S., Christogiorgos, S., Deligeoroglou, E., Christopolos, P., Tsiantis, J., \& Creatsas, G. (2009). Anxiety and depression in adolescents with polycystic ovary syndrome and MayerRokitansky-Kuster-Hauser syndrome. Journal of Psychosomatic Obstetrics and Gynecology, 30, 83-88.

Lee, J. S. (2015). It's not just physical: The adverse psychological effects of polycystic ovary syndrome in adolescents. Women's Healthcare: A Clinical Journal for NPs, 3, 20-27.

Linehan, M. M. (2015). DBT skills training manual (2nd ed.). New York, NY: Guilford Press.

Mahoney, D. (2014). Lifestyle modification intervention among infertile overweight and obese women with polycystic ovary syndrome. Journal of the American Association of Nurse Practitioners, 26, 301-308. doi:10.1002/2327-6924.12073

Myers, J. E., \& Sweeney, T. J. (2005). The Indivisible Self: An evidence-based model of wellness [Reprint]. The Journal of Individual Psychology, 61, 269-279. 
Myers, J. E., Sweeny, T. J., \& Witmer, J. M. (2000). The Wheel of Wellness counseling for wellness: A holistic model for treatment planning. Journal of Counseling \& Development, 78, 251-266. doi:10.1002/i.1556-6676.2000.tb01906.x

Rofey, D. L., Szigethy, E. M., Noll, R. B., Dahl, R. E., Lobst, E., \& Arslanian, S. A. (2009). Cognitivebehavioral therapy for physical and emotional disturbances in adolescents with polycystic ovary syndrome. Journal of Pediatric Psychology, 34, 156-163. doi:10.1093/ipepsy/isn057

Smith, J. W., \& Taylor, J. S. (2011). Polycystic ovary syndrome: Evidence-based strategies for managing symptoms and preventing long-term sequelae. Nursing for Women's Health, 15, 404-411. doi:10.1111/j.1751-486X.2011.01664.x

Snyder, B. S. (2005). Polycystic ovary syndrome (PCOS) in the adolescent patient: Recommendations for practice. Pediatric Nursing, 31, 416-421.

Sweeney, T. J., \& Myers, J. E. (2004). Counseling for wellness. In J. E. Myers \& T. J. Sweeney (Eds.), Counseling for wellness: Theory, research, and practice (pp. 185-196). Alexandria, VA: American Counseling Association.

Tehrani, F. R. (2015). Polycystic ovary syndrome: An apparently simple yet challenging diagnosis. International Journal of Endocrinology and Metabolism, 13, 1-2. doi:10.5812/ijem.28557v2 